\title{
ON THE LINEAR PARTIAL $q$-DIFFERENCE EQUATION OF GENERAL TYPE*
}

BY

\section{RAYMOND ADAMS}

Introduction. In a paper published some time agot the author defined the linear partial $q$-difference equation and examined a class of such equations. The general methods employed were those introduced by Birkhoff $\ddagger$ in studying the linear ordinary difference equation. It is our purpose here to investigate the general problem of the partial $q$-difference equation, to which the methods of Birkhoff, based primarily on iteration, are not applicable. We propose to establish existence theorems by direct convergence proofs.

The equation considered is $\S$

$$
\sum_{i, j=0}^{n} a_{i j}(x, y) f\left(q^{i} x, r^{j} y\right)=0,
$$

in which the $a_{i j}(x, y)$ are known functions of the complex variables $x$ and $y$, analytic at the place $(0,0) ; q$ and $r$ are constants, not zero and of moduli $\neq 1$; and $f(x, y)$ is the function to be determined. We may write

$$
\begin{aligned}
a_{i j}(x, y)=a_{i j 00}+a_{i j 10} x+a_{i j 01} y+\cdots & \text { for }|x|<R_{1}, \\
& |y|<R_{2} \quad(i, j=0,1, \cdots, n),
\end{aligned}
$$

and without loss of generality we may and do assume $|q|$ and $|r|$ both greater than 1.

For convenience in notation we define

$$
\begin{aligned}
& g(\rho, \sigma, k, l, m, p)=\sum_{i, j=0}^{n} a_{i j m p} \rho^{i} q^{k i} \sigma^{i} r^{l j}, \\
& g_{\diamond 0}(\rho, \sigma, k, l, m, p)=\sum_{i, j=0}^{n} i^{\bullet} a_{i j m p} \rho^{i} q^{k i} \sigma^{i} r^{l j}, \\
& g_{0 s}(\rho, \sigma, k, l, m, p)=\sum_{i, j=0}^{n} j^{s} a_{i j m p} \rho^{i} q^{k i \sigma_{r} l j} \quad(s=1,2, \cdots) .
\end{aligned}
$$

* Presented to the Society, September 7, 1928; received by the editors August 24, 1928.

$\dagger$ Adams, The general theory of a class of linear partial q-difference equations, these Transactions, vol. 26 (1924), pp. 283-312; this paper will hereafter be referred to as I.

$\ddagger$ Birkhoff, General theory of linear difference equations, these Transactions, vol. 12 (1911), pp. 243-284.

$\S$ It is clearly possible to write equation (B) of $I$ in this form. 
Then the algebraic equation

$$
g(\rho, \sigma, 0,0,0,0)=0
$$

is called the characteristic equation of (1) for $(0,0)$. We speak of a pair of values $\rho_{0}, \sigma_{0}$ satisfying (4) as the characteristic number-pair $\left(\rho_{0}, \sigma_{0}\right)$; it is said to be simple if $g\left(\rho, \sigma_{0}, 0,0,0,0\right)=0$ has $\rho_{0}$ as a simple root and if $g\left(\rho_{0}, \sigma\right.$, $0,0,0,0)=0$ has $\sigma_{0}$ as a simple root.

We term $\left(\rho_{0}, \sigma_{0}\right)$ a regular characteristic number-pair when the following assumptions are fulfilled: (a) both $\rho_{0}$ and $\sigma_{0}$ are finite and different from zero; (b) $\left(\rho_{0}, \sigma_{0}\right)$ is simple and satisfies none of the equations

$$
g(\rho, \sigma, k, l, 0,0)=0,
$$

where $k$ and $l$ are positive integers or zero but not both zero; (c) $a_{n n 00}$ is not zero; (d) $\rho_{0}$ satisfies none of the equations

$$
\sum_{i=0}^{n} a_{i n 00 \rho^{i} q^{k i}}=0 \quad(k=0,1, \cdots, K-1) ;
$$

(e) $\sigma_{0}$ satisfies none of the equations

$$
\sum_{j=0}^{n} a_{n j 00 \sigma^{i} r^{l i}}=0 \quad(l=0,1, \cdots, L-1) .
$$

$K$ and $L$ are integers presently to be determined.

We direct our attention first, in $\$ \S 1,2$, to the case of a regular characteristic number-pair, exhibiting a corresponding series that formally satisfies (1) and by proof of convergence demonstrating the existence of a solution analytic in the vicinity of $(0,0)$. In $\$ \S 3,4$ we examine, with similar results, the case of an irregular characteristic number-pair whose irregularity is due to the failure of assumption (b) alone. $\$ 5$ is devoted to the cases of other irregular characteristic number-pairs. We conclude in $\$ 6$ with a brief consideration of the non-homogeneous equation obtained by adding to the lefthand member of (1) a known function.

It is of interest and importance to observe (i) that $\$ \S 1,2$ provide a simple and brief derivation of the fundamental existence theorem of $\$ 2$ of I; (ii) that if the coefficient functions $a_{i j}(x, y)$ are analytic at the place $(\infty, \infty)$, or at the place $(0, \infty)$, or at the place $(\infty, 0)$, the situation with regard to solutions in the vicinity of this place is entirely parallel to that described in detail here; and (iii) that the methods employed here are applicable at once to the linear partial $q$-difference equation of general type in $N$ independent variables. 
1. Regular characteristic number-pair; formal series. Corresponding to a regular characteristic number-pair $\left(\rho_{0}, \sigma_{0}\right)$ there exists a formal solution of (1),

$$
s(x, y)=x^{a} y^{b}\left(s_{00}+s_{10} x+s_{01} y+\cdots\right),
$$

where*

$$
a=\frac{\log \rho_{0}}{\log q} \text { and } b=\frac{\log \sigma_{0}}{\log r} .
$$

The coefficients in (6) may be calculated by substitution of the series in (1); $s_{00}$ is arbitrary, but the subsequent coefficients are determined uniquely in terms of it by the relation

$$
s_{k l}=-\frac{\sum_{i=0}^{k-1} s_{i l} g\left(\rho_{0}, \sigma_{0}, i, l, k-i, 0\right)+\sum_{i=0}^{k} \sum_{j=0}^{l-1} s_{i j} g\left(\rho_{0}, \sigma_{0}, i, j, k-i, l-j\right)}{g\left(\rho_{0}, \sigma_{0}, k, l, 0,0\right)} .
$$

It may be remarked that assumptions (c), (d), and (e) are not needed for this section.

2. Regular characteristic number-pair; existence of an analytic solution. For convenience we assume that the associated radii of convergence of the series (2) are all greater than 1 . It can readily be shown, as in the case of the linear ordinary $q$-difference equation, that this hypothesis is no real restriction. $\dagger$

We seek to prove the convergence of the series (6) by finding a convergent dominant series. Let us first examine the denominator of (7) with a view to establishing the inequality

$$
\left|g\left(\rho_{0}, \sigma_{0}, k, l, 0,0\right)\right|>C\left|q^{n(a+k)} \boldsymbol{r}^{n(b+l)}\right| \quad(C>0)
$$

for $k, l=0,1, \cdots$ but not both zero. After the factor $\left|q^{n(a+k)} r^{n(b+l)}\right|$ is removed from $\left|g\left(\rho_{0}, \sigma_{0}, k, l, 0,0\right)\right|$, the remaining factor is

$$
\begin{aligned}
& \mid a_{n n 00}+\frac{a_{n, n-1,00}}{r^{b+l}}+\cdots+\frac{a_{n 000}}{r^{n(b+l)}}+\frac{a_{n-1, n 00}}{q^{a+k}}+\frac{a_{n-1, n-1,00}}{q^{a+k} r^{b+l}}+\cdots \\
& \quad+\frac{a_{n-1,000}}{q^{a+b} r^{n(b+l)}}+\cdots+\frac{a_{0 n 00}}{q^{n(a+k)}}+\frac{a_{0, n-1,00}}{q^{n(a+k)} r^{b+l}}+\cdots+\frac{a_{0000}}{q^{n(a+k)} r^{n(b+l)}} \mid
\end{aligned}
$$

* Throughout the paper we take, for definiteness, those determinations of $\log x, \log y, \log \rho_{0}$, $\log \sigma_{0}, \log q$, and $\log r$ in which the coefficient of $(-1)^{1 / 2}$ is positive or zero and less than $2 \pi$.

$\dagger$ Adams, On the linear ordinary q-difference equation, Annals of Mathematics, (2), vol. 30 (1929), No. 2 ; we shall refer to this paper as II. 
Since $a_{n n 00}$ is not zero, a value of $l$, which we denote by $L$, can be found to make

$$
\left|\frac{a_{n, n-1,00}}{r^{b+l}}+\cdots+\frac{a_{n 000}}{r^{n(b+l)}}\right|<\frac{\left|a_{n n 00}\right|}{n+2} \text { for } l \geqq L .
$$

Then we have

$$
\begin{aligned}
\mid a_{i n 00}+\frac{a_{i, n-1,00}}{r^{b+l}} & +\cdots+\frac{a_{i 000}}{r^{n(b+l)}}|\leqq| a_{i n 00}|+| \frac{a_{i, n-1,00}}{r^{b+L}} \mid \\
& +\cdots+\left|\frac{a_{i 000}}{r^{n(b+L)}}\right| \text { for } l \geqq L \quad(i=0,1, \cdots, n-1),
\end{aligned}
$$

and a value of $k$, which we denote by $K$, can be found to yield the inequality

$$
\begin{aligned}
& \frac{\left|a_{i n 00}\right|+\left|\frac{a_{i, n-1,00}}{r^{b+L}}\right|+\cdots+\left|\frac{a_{i 000}}{r^{n(b+L)}}\right|}{\left|q^{(n-i)(a+k)}\right|}<\frac{\left|a_{n n 00}\right|}{n+2} \text { for } k \geqq K, \quad l \geqq L \\
& (i=0,1, \cdots, n-1) .
\end{aligned}
$$

Thus for $k \geqq K, l \geqq L$ the quantity (9) is greater than $\left|a_{n n 00}\right| /(n+2)$.

For any particular $l<L$ we can, by virtue of assumption (e), choose $k_{l}$ so large that the sum of terms containing $q$ in (9) is in absolute value less than

$$
\frac{1}{2}\left|a_{n n 00}+\frac{a_{n, n-1,00}}{r^{b+l}}+\cdots+\frac{a_{n 000}}{r^{n(b+l)}}\right| \text { for } k \geqq k_{l},
$$

and the quantity (9) is then greater than (10).

For any particular $k<K$ we can, because of assumption (d), choose $l_{k}$ so large that the sum of terms containing $r$ in (9) is in absolute value less than

$$
\frac{1}{2}\left|a_{n n 00}+\frac{a_{n-1, n 00}}{q^{a+k}}+\cdots+\frac{a_{0 n 00}}{q^{n(a+k)}}\right| \text { for } l \geqq l_{k},
$$

and the quantity (9) is then greater than (11).

Now there are only a finite number of pairs of values $k, l$ not included in the three classes $k \geqq K, l \geqq L ; k<K, l \geqq l_{k} ; l<L, k \geqq k_{l}$. For each of these pairs, by assumption (b), the quantity (9) has a positive value $C_{k l}$. If then we take for $C$ the smallest of the constants

$\frac{\left|a_{n n 00}\right|}{n+2} ;(10)$ for $l=0,1, \cdots, L-1 ;(11)$ for $k=0,1, \cdots, K-1 ; C_{k l}$, the inequality (8) will be satisfied. 
Turning to the numerator of (7) and recalling that the series (2) converge for $x=y=1$, we have

$$
\left|a_{i j m p}\right|<M^{\prime} \quad(i, j=0,1, \cdots, n ; m, p=0,1, \cdots),
$$

$M^{\prime}$ being a suitable positive number. Let the largest of the quantities $\left|q^{i a} r^{i b}\right|(i, j=0,1, \cdots, n)$ be denoted by $M^{\prime \prime}$. Since $|q|$ and $|r|$ are greater than 1 , we then have

Defining $\bar{s}_{00}$ as $\left|s_{00}\right|$ and setting

$$
\begin{aligned}
\left|s_{k l}\right|<\frac{1}{C\left|q^{n(a+k)} r^{n(b+l)}\right|} & {\left[( n + 1 ) ^ { 2 } M ^ { \prime } M ^ { \prime \prime } | q ^ { n k } r ^ { n l } | \left(\sum_{i=0}^{k-1}\left|s_{i l}\right|\right.\right.} \\
& \left.\left.+\sum_{i=0}^{k}\left(\left|s_{i 0}\right|+\left|s_{i 1}\right|+\cdots+\left|s_{i, l-1}\right|\right)\right)\right] .
\end{aligned}
$$

$$
M=\frac{(n+1)^{2} M^{\prime} M^{\prime \prime}}{C\left|q^{n a} r^{n b}\right|}
$$

we obtain the following bound for $s_{k l}$ :

$$
\bar{s}_{k l}=M\left[\sum_{i=0}^{k-1}\left(\bar{s}_{i 0}+\bar{s}_{i 1}+\cdots+\bar{s}_{i l}\right)+\bar{s}_{k 0}+\bar{s}_{k 1}+\cdots+\bar{s}_{k, l-1}\right],
$$

$k$ and $l$ being any positive integers or zero but not both zero. From this formula follows at once the recurrence relation

$$
\bar{s}_{k l}=M \sum_{i=0}^{k-1} \bar{s}_{i l}+(M+1) \bar{s}_{k, l-1},
$$

in which $\bar{s}_{k,-1}$ and $\bar{s}_{-1, l}$ are to be interpreted as zero.

It is possible to show directly that the series

$$
\sum_{k, l=0}^{\infty} \bar{s}_{k l} x^{k} y^{l}
$$

has associated radii of convergence greater than zero. For simplicity and for convenience in our later work, however, we prefer to establish this fact indirectly, by proving the same property for the dominant series

$$
\sum_{k, l=0}^{\infty} S_{k l} x^{k} y^{l},
$$

where $S_{00}=\bar{s}_{00}$ and $S_{k l}$ is determined by the recurrence formula

$$
S_{k l}=(M+1)\left[\sum_{i=0}^{k-1} S_{i l}+S_{k, l-1}\right] \quad\left(S_{k,-1}=S_{-1, l}=0\right) .
$$

The basis of our proof is the rather obvious 
LemMa. A sufficient condition that a power series in two variables with positive coefficients have associated radii of convergence greater than zero is that the simple power series which it becomes upon setting $y=x$ have a positive radius of convergence.

From (16) we have*

$$
S_{k l} \leqq(M+1)\left(S_{k-1, l}+S_{k, l-1}\right)+S_{k-1, l} \leqq(M+2)\left(S_{k-1, l}+S_{k, l-1}\right) .
$$

The series (15) is therefore dominated by

$$
T(x, y)=\sum_{k, l=0}^{\infty} T_{k l} x^{k} y^{l}
$$

in which $T_{00}=S_{00}$ and $T_{k l}$ is given by the relation

$$
T_{k l}=(M+2)\left(T_{k-1, l}+T_{k, l-1}\right) \quad\left(T_{k,-1}=T_{-1, l}=0\right) .
$$

In the simple power series

the coefficients are

$$
T(x, x)=\sum_{\nu=0}^{\infty} T, x^{\nu}
$$

$$
T_{\nu}=\sum_{k+l=\nu} T_{k l}=2(M+2) \sum_{k+l=\eta-1} T_{k l}=2(M+2) T_{\nu-1} .
$$

Hence $T(x, x)$ converges for $|x|<1 /[2(M+2)]$ and the series (17), (15), (14), and (6) all have associated radii of convergence that are positive. Thus we obtain

THEOREM I. Corresponding to each regular characteristic number-pair $\left(\rho_{0}, \sigma_{0}\right)$ there exists a solution of the equation (1) which is analytic in the vicinity of the place $(0,0)$ and is expressed there by the series $(6)$.

It may be added that if $a_{i n}(x, y)\left[a_{n i}(x, y)\right]$ vanishes identically for $i=0,1, \cdots, n-1$, the region of analyticity of the solutions can be extended away from the origin in the $x$-plane [y-plane] by repeated use of the equation (1) itself; if both of these conditions are satisfied, each of the series (6) converges out to the nearest place which is a singularity of the coefficient functions $a_{i j}(x, y)$ or a zero of $a_{n n}(x, y)$ and represents an analytic solution of (1) thus far out from $(0,0)$; if, further, the $a_{i j}(x, y)$ are all rational functions, these solutions analytic

* The proof of convergence of (15), as originally constructed by the author, is based on a comparison of $\sum_{k+l=\nu} S_{k l}$ with $S_{60}(1+1+1)^{\gamma}$, by which it is dominated. The particularly simple discussion given in this paragraph follows the lines of a suggestion by J. D. Tamarkin. 
near $(0,0)$ are analytic except for poles over the entire finite $x$-and $y$-planes, except perhaps for $x=0$ and $y=0$.

3. Irregular characteristic number-pair, the irregularity arising from failure of assumption (b) only; formal series. Let us consider first a characteristic number-pair $\left(\rho_{0}, \sigma_{0}\right)$ which is of multiplicity $\mu(\geqq 1)$ in $\rho$ (i.e., $g\left(\rho, \sigma_{0}\right.$, $0,0,0,0)=0$ has $\rho_{0}$ as a root of multiplicity $\mu$ ) and which satisfies exactly $\nu(\geqq 0)$ of the equations $(5)$, each equation being counted a number of times equal to the multiplicity in $\rho$ of the solution $\left(\rho_{0}, \sigma_{0}\right)$. To this number-pair there correspond, whether $\left(\rho_{0}, \sigma_{0}\right)$ is simple or multiple in $\sigma, \mu$ formal solutions of (1),

$$
s_{i}(x, y)=x^{a} y^{b}\left[(\text { P.S. })+t(\text { P.S. })+\cdots+t^{\nu+i-1}(\text { P.S. })\right] \quad(i=1,2, \cdots, \mu),
$$

where

$$
a=\frac{\log \rho_{0}}{\log q}, \quad b=\frac{\log \sigma_{0}}{\log r}, \quad t=\frac{\log x}{\log q},
$$

and (P.S.) is used in a generic sense to denote a power series in $x$ and $y$. The coefficients in (18) may be calculated by substitution of the series in(1). In the power series in $s_{i}(x, y)$ there are in general ${ }^{*} \nu+i$ coefficients whose values may be assigned arbitrarily; all of the other coefficients are then uniquely dətermined.

It is now quite evident from symmetry that if $\left(\rho_{0}, \sigma_{0}\right)$ is of multiplicity $\mu$ in $\sigma$ and satisfies exactly $\nu$ of the equations (5), each equation being counted a number of times equal to the multiplicity in $\sigma$ of the solution $\left(\rho_{0}, \sigma_{0}\right)$, there correspond, whether $\left(\rho_{0}, \sigma_{0}\right)$ is simple or multiple in $\rho, \mu$ formal solutions precisely like (18) except that $t$ is replaced by

$$
\tau=\frac{\log y}{\log r} .
$$

The two foregoing paragraphs suggest an interesting question. One might rather naturally expect, if $\nu$ is zero and if $\left(\rho_{0}, \sigma_{0}\right)$ is of multiplicity $\mu_{1}$ in $\rho$ and of multiplicity $\mu_{2}$ in $\sigma$, that there should be $\mu_{1} \cdot \mu_{2}$ solutions corresponding to this number-pair. Only $\mu_{1}+\mu_{2}-1$ independent solutions, however, have been exhibited here. Are there other solutions, and if so of what form are they?

A second point of special interest arises when $\nu$ is $\geqq 1$. Let us suppose, for simplicity, that $\left(\rho_{0}, \sigma_{0}\right)$ is simple; then one might expect only one formal solution corresponding to it. Yet the condition (5) does not favor $x$ and $q$

* Únder special conditions there may be more. 
over $y$ and $r$, and it is fairly clear that there must be a second independent solution like (18) except for the replacement of $t$ by $\tau$. In fact not only is this true but it can readily be verified also that in general there are still other independent formal solutions containing powers of both $t$ and $\tau$,

$$
\left.x^{a} y^{b}\left[(\text { P.S. })+t(\text { P.S. })+\cdots+t^{\nu_{1}} \text { (P.S. }\right)+\tau(\text { P.S. })+\cdots+\tau^{\nu_{2}} \text { (P.S.) }\right] \text {. }
$$

If $\left(\rho_{0}, \sigma_{0}\right)$ is multiple, the situation is analogous.

As in $\$ 1$, the assumptions (c), (d), and (e) are not needed for this section.

4. Irregular characteristic number-pair, the irregularity arising from failure of assumption (b) only; existence of analytic solutions. We consider in detail the case in which the equation (1) is satisfied formally by the series (18). Let us examine

$$
\begin{aligned}
s_{1}(x, y)= & x^{a} y^{b}\left[\left(s_{00}^{(\nu)}+s_{10}^{(\nu)} x+s_{01}^{(\nu)} y+\cdots\right)+t\left(s_{00}^{(\nu-1)}+s_{10}^{(\nu-1)} x\right.\right. \\
& \left.\left.+s_{01}^{(\nu-1)} y+\cdots\right)+\cdots+t^{\nu}\left(s_{00}^{(0)}+s_{10}^{(0)} x+s_{01}^{(0)} y+\cdots\right)\right],
\end{aligned}
$$

assuming for the sake of generality that $\nu$ is positive; the methods employed are then clearly applicable to $s_{i}(x, y)(i=2,3, \cdots, \mu)$. As in $\$ 2$ we make no real restriction in assuming the associated radii of convergence of all the series (2) greater than 1.

In order to prove the power series in $s_{1}(x, y)$ convergent in the vicinity of $(0,0)$ we observe first that the coefficients $s_{k l}^{(m)}$ in these series satisfy the following relations (cf. (3)):

$$
\begin{aligned}
s_{k l}^{(m)}= & -\left\{\sum_{i=0}^{k-1} s_{i l}^{(m)} g\left(\rho_{0}, \sigma_{0}, i, l, k-i, 0\right)+\sum_{i=0}^{k} \sum_{j=0}^{l-1} s_{i j}^{(m)} g\left(\rho_{0}, \sigma_{0}, i, j, k-i, l-j\right)\right. \\
& +\sum_{i=0}^{k} \sum_{j=0}^{l}\left[\left(\begin{array}{c}
\nu-m+1 \\
1
\end{array}\right) s_{i j}^{(m-1)} g_{10}\left(\rho_{0}, \sigma_{0}, i, j, k-i, l-j\right)\right. \\
& +\left(\begin{array}{c}
\nu-m+2 \\
2
\end{array}\right) s_{i j}^{(m-2)} g_{20}\left(\rho_{0}, \sigma_{0}, i, j, k-i, l-j\right)+\cdots \\
& \left.\left.+\left(\begin{array}{c}
\nu \\
m
\end{array}\right) s_{i j}^{(c)} g_{m 0}\left(\rho_{0}, \sigma_{0}, i, j, k-i, l-j\right)\right]\right\} \div g\left(\rho_{0}, \sigma_{0}, k, l, 0,0\right) \\
& (m=0,1, \cdots, \nu) .
\end{aligned}
$$

Whenever $g\left(\rho_{0}, \sigma_{0}, k, l, 0,0\right)$ vanishes, the numerator of this quotient is of course also zero. Bounds for the quantities $s_{k l}^{(m)}$ are determined here as they were for the $s_{k l}$ in $\S 2$. We let $M^{\prime}$ and $M^{\prime \prime}$ have the same significance as before, let $B$ stand for the largest of the binomial coefficients appearing in any one of the expressions (21), and redefine $M$ as follows: 


$$
M=\frac{(n+1)^{2} n^{\nu} B M^{\prime} M^{\prime \prime}}{C\left|q^{n a} r^{n b}\right|}
$$

Thus, defining $\bar{s}_{00}^{(0)}$ as $\left|s_{00}^{(0)}\right|$ and in fact $\bar{s}_{k l}^{(m)}$ whenever $s_{k l}^{(m)}$ is arbitrary as $\left|s_{k l}^{(m)}\right|$, we obtain a bound for (21):

$$
\begin{aligned}
\bar{s}_{k l}^{(m)}=M\left[\sum_{i=0}^{k-1} \bar{s}_{i l}^{(m)}+\right. & \sum_{i=0}^{k} \sum_{j=0}^{l-1} \bar{s}_{i j}^{(m)} \\
& \left.+\sum_{i=0}^{k} \sum_{j=0}^{l}\left(\bar{s}_{i j}^{(m-1)}+\bar{s}_{i j}^{(m-2)}+\cdots+\bar{s}_{i j}^{(0)}\right)\right] .
\end{aligned}
$$

For $m=0$ the right-hand member reduces precisely to that of (12); we therefore infer at once the convergence, in the vicinity of $(0,0)$, of the last power series in (20). For $m=1$ equation (22) becomes

$$
s_{k l}^{(1)}=M\left[\sum_{i=0}^{k-1} \bar{s}_{i l}^{(1)}+\sum_{i=0}^{k} \sum_{j=0}^{l-1} \bar{s}_{i j}^{(1)}+\sum_{i=0}^{k} \sum_{j=0}^{l} \bar{s}_{i j}^{(0)}\right] .
$$

Taking account of the obvious relations $\bar{s}_{i j}^{(0)} \leqq \bar{s}_{i j}^{(1)} \leqq \cdots \leqq \bar{s}_{i j}^{\nu}$, we have

$$
\begin{aligned}
\bar{s}_{k l}^{(1)} & =M \sum_{i=0}^{k-1} \bar{s}_{i l}^{(1)}+M \sum_{i=0}^{k} \bar{s}_{i l}^{(0)}+(M+1) \bar{s}_{k, l-1}^{(1)} \\
& =M \sum_{i=0}^{k-1}\left(\bar{s}_{i l}^{(1)}+\bar{s}_{i l}^{(0)}\right)+M\left[M \sum_{i=0}^{k-1} \bar{s}_{i l}^{(0)}+(M+1) \bar{s}_{k, l-1}^{(0)}\right]+(M+1) \bar{s}_{k, l-1}^{(1)} \\
& <(M+1)^{2}\left[\sum_{i=0}^{k-1} \bar{s}_{i l}^{(1)}+\bar{s}_{k, l-1}^{(1)}\right]=S_{k l}^{(1)} .
\end{aligned}
$$

The $S_{k l}^{(1)}$, thus defined by a recurrence relation exactly like (16); are the coefficients of a power series convergent in the vicinity of $(0,0)$; we therefore infer the same property for the next to the last series in (20). It is now easy to establish by induction the inequality

$$
s_{k l}^{(j)}<(M+1)^{i+1}\left[\sum_{i=0}^{k-1} \bar{s}_{i l}^{(j)}+\bar{s}_{k, l-1}^{(j)}\right],
$$

frcm which follows at once the convergence, in the vicinity of $(0,0)$, of all th : remaining power series in (20).

If there correspond to $\left(\rho_{0}, \sigma_{0}\right)$ series like (18) except for the replacement of $t$ by $\tau$, or series of the type (19), their convergence may be proved in like manner; in each of these cases the $g_{0_{s}}\left(\rho_{0}, \sigma_{0}, k, l, m, p\right)$ (cf. (3)) play a rôle like that of $g_{\bullet 0}\left(\rho_{0}, \sigma_{0}, k, l, m, p\right)$ above. Hence we conclude 
ThEOREM II. Corresponding to each irregular characteristic number-pair $\left(\rho_{0}, \sigma_{0}\right)$ whose irregularity is due to the failure of assumption (b) alone, there exist a certain number of solutions of the equation (1) which are analytic in the vicinity of the place $(0,0)$ and are expressed there by the series exhibited in $\$ 3$.

The italicized additional statements following Theorem I also apply here.

To complete the discussion of this and the preceding section we observe that $g\left(\rho_{0}, \sigma_{0}, k, l, 0,0\right)$ can vanish for at most a finite number of pairs of values $k, l$ when assumption (b) alone fails. This follows immediately since (i) for $k \geqq K$ and $l \geqq L, g\left(\rho_{0}, \sigma_{0}, k, l, 0,0\right)$ is not zero; and (ii) for any particular $k[l]$ less than $K[L], g\left(\rho_{0}, \sigma_{0}, k, l, 0,0\right)$ is a polynomial of degree $n$ in $r^{l}\left[q^{k}\right]$ and so can vanish for only a finite number of values of $l[k]$. Therefore only series with a finite number of logarithmic terms can occur.

5. Other irregular characteristic number-pairs. It is clear that in certain cases of irregular characteristic number-pairs due to the failure of assumptions (a) or (c) the method employed by the author in II can be used to bring the equation (1) into a form to which the work of the preceding sections is applicable; these cases occur when the coefficients (2) are of the form $a_{i j}(x, y)=x^{\alpha_{i j} y_{i j}}$. [convergent power series in $x$ and $y$ ] $(i, j=0,1, \cdots, n)$, the $\alpha_{i j}$ and $\beta_{i j}$ being integers $\geqq 0$.

Let $a_{i}(i=0,1, \cdots, n)$ denote the least of the $n+1$ exponents $\alpha_{i j}(j=0$, $1, \cdots, n)$; similarly let $b_{j}(j=0,1, \cdots, n)$ represent the least of the exponents $\beta_{i j}(i=0,1, \cdots, n)$. First consider the case of $b_{0}=b_{n}=0$. We plot the points $a_{i}$ in the $i a$-plane and draw a broken line above or upon which all these points lie and of which each vertex and end point is a point of the set. Then to a segment of slope $\mu^{\prime}$ there corresponds in general an appropriate number of formal solutions like (6) or those of $\$ 3$ except for the additional factor

$$
q^{\left(\mu^{\prime} / 2\right)\left(t^{2}-t\right)} \text {. }
$$

Secondly, in the case of $a_{0}=a_{n}=0$ the situation is entirely parallel, the additional factor in the solution being

$$
r^{\left(\nu^{\prime} / 2\right)\left(r^{2}-r\right)} \text {. }
$$

If neither of these conditions, $b_{0}=b_{n}=0$ and $a_{0}=a_{n}=0$, is satisfied, a combination of the two processes here indicated may be used, the solutions obtained containing factors of both types (23) and (24). The solutions can be shown to converge in the vicinity of $(0,0)$ if after subjecting $(1)$ to the transformation 


$$
f(x, y)=q^{\left(\mu^{\prime} / 2\right)\left(t^{2}-t\right)} r^{\left(\nu^{\prime} / 2\right)\left(r^{2}-r\right)} g(x, y),
$$

the resulting equation has a characteristic equation whose solutions satisfy assumptions (a), (c), (d), and (e).

It is quite clear that assumptions (c), (d), and (e) are essential to the convergence proofs only, not to the existence of formal solutions. In this connection we may point out that the present work sheds a certain amount of light upon the cases which gave rise to the question proposed at the close of $\$ 2$ of I; under the definitions set forth here we have in these cases an irregularity, due to the failure of assumption (c).

Reference should also be made in this section to the method of treating irregularities which has been elaborated in $\$ 3$ of I and which is sometimes efficacious.

We shall not attempt here to answer the question as to what is the most general solution of the equation (1). It may be remarked, however, that a linear combination of any finite number of particular solutions, in which the coefficients are arbitrary functions $p_{i}(x, y)$ satisfying the conditions

$$
p_{i}(q x, y)=p_{i}(x, r y)=p_{i}(x, y),
$$

is also a solution.

6. The non-homogeneous equation. In this section we consider briefly the equation obtained by adding to the left member of (1) a known function $b(x, y)$ of the same character as the $a_{i j}(x, y)$ :

$$
\sum_{i, j=0}^{n} a_{i j}(x, y) f\left(q^{i} x, r^{j} y\right)+b(x, y)=0 .
$$

For this equation we set forth

TheOREM III. If among the number-pairs $\left(q^{i}, r^{j}\right)(i, j=0,1, \cdots)$ there are exactly $p_{1}$ which satisfy the equation (3), each being counted according to its multiplicity in $\rho$, the equation (26) has a formal solution

$$
\text { (P.S.) }+t(\text { P.S. })+\cdots+t^{p_{1}} \text { (P.S.) }
$$

in which the coefficients of the power series may be calculated by substitution in (26); if exactly $p_{2}$ of these number-pairs satisfy (3) when each is counted according to its multiplicity in $\sigma$, there is a second formal solution

$$
(\text { P.S. })+\tau(\text { P.S. })+\cdots+\tau^{p_{2}} \text { (P.S.) ; }
$$

there may also be other formal solutions involving $t$ and $\tau$ simultaneously. If assumption (c) is satisfied and if we have also 


$$
\begin{array}{ll}
\sum_{i=0}^{n} a_{i n 00} q^{k i} \neq 0 & \left(k=0,1, \cdots, K^{\prime}-1\right), \\
\sum_{i=0}^{n} a_{n j 00} r^{l j} \neq 0 & \left(l=0,1, \cdots, L^{\prime}-1\right),
\end{array}
$$

where $K^{\prime}$ and $L^{\prime}$ are determined in the same manner as were $K$ and $L$, the methods of $\$ \$ 2,4$ may be employed to prove that each formal solution converges in the vicinity of $(0,0)$ and so represents an analytic solution there.

It is clear that the sum of a particular solution of (26) and a linear combination of any finite number of solutions of the associated homogeneous equation (1), with arbitrary coefficients $p_{i}(x, y)$ satisfying the condition (25), is also a solution of (26).

BroWn UNIVERSITY, Providence, R. I. 